\title{
Continuités et ruptures
}

De Techniques \& culture à... Techniques \& culture

Frédéric Joulian et Robert Cresswell

\section{CpenEdition}

Journals

Édition électronique

URL : https://journals.openedition.org/tc/2172

DOI : $10.4000 /$ tc. 2172

ISSN : 1952-420X

Éditeur

Éditions de l'EHESS

Édition imprimée

Date de publication : 12 décembre 2007

ISSN : 0248-6016

Référence électronique

Frédéric Joulian et Robert Cresswell, «Continuités et ruptures », Techniques \& Culture [En ligne], 48-49 | 2007, mis en ligne le 28 août 2008, consulté le 29 septembre 2022. URL : http://

journals.openedition.org/tc/2172 ; DOI : https://doi.org/10.4000/tc.2172

Ce document a été généré automatiquement le 29 septembre 2022.

Tous droits réservés 


\title{
Continuités et ruptures
}

\author{
De Techniques \& culture à... Techniques \& culture
}

Frédéric Joulian et Robert Cresswell

1 Avec la livraison de ce numéro double de Techniques \& culture, la revue souhaite informer ses lecteurs de la nouvelle ligne éditoriale que nous inaugurerons avec le numéro suivant. Pour un temps, le label "nouvelle série ", marquera tout à la fois la continuité axiomatique avec la revue telle qu'elle s'est développée depuis le début des années 1980 et le renouveau formel que nous engageons dans une action que nous espérons structurante et au long cours. L'objectif général est bien sûr de rendre compte des rapports entre « technique et culture, techniques et cultures, technique et cultures, techniques et culture ", et, plus sérieusement, de rapporter et structurer l'extrême diversité des recherches attentives à la matérialité des activités humaines dans le temps et l'espace.

2 Ce numéro intermédiaire entre la formule précédente et la nouvelle, nous permet d'œuvrer à la mise en place d'une nouvelle architecture (principalement thématique) et d'un nouveau fonctionnement dont nous verrons les fruits avec les numéros de l'année 2009. En attendant ces nouvelles productions, nous souhaitons rendre compte ici d'un certain nombre de changements opérés au cours de l'année 2007. La direction de la revue a élu un nouveau rédacteur en chef, Frédéric Joulian, succédant à Jean-Luc Jamard, arrivé au terme de son mandat en 2006. Parallèlement à cela, la rédaction a déménagé de Paris à Marseille au sein de l'unité de recherches « Sociologie, histoire et anthropologie des dynamiques culturelles" (EHESS- CNRS), au Centre de la Vieille Charité. La nouvelle formule sera mise en place dans les prochains numéros. Elle est le résultat d'un travail -souvent invisible- d'une communauté de chercheurs discrets mais actifs que l'on ne mentionne généralement pas, mais sans laquelle les revues scientifiques ne pourraient correctement fonctionner.

3 Le comité de rédaction est en cours de restructuration et se voit rééquilibré vers de nouvelles disciplines (sociologie, histoire de l'art, communication, muséologie) qui invitent la publication à une ouverture thématique plus large, mais également, à un équilibre disciplinaire qui s'était quelque peu défait au fil des ans. 
4 Les différentes opérations administratives (récupération des textes, déménagement, concours, recrutement de personnels, aménagement du bureau, recherche de nouveaux financements) pour développer une nouvelle maquette furent laborieux. L'installation de la revue devrait cependant s'achever dans les semaines à venir. L'écart important entre la livraison du numéro 46-47 (made in Paris) et du numéro 48-49 (made in Marseille) s'explique par des changements administratifs importants qui sont heureusement en voie d'achèvement. Nous tenions à nous en excuser auprès des lecteurs. Le bon fonctionnement d'une revue exige la présence de personnel compétent que nous avons recruté au cours de l'année 2007. Le nouveau secrétariat de rédaction s'est engagé avec enthousiasme sur ce projet de refonte et de développement de la revue et nous tenons dès à présent à l'en remercier.

5 Les grandes lignes directrices de réorganisation développées ci-dessous devraient permettre tout à la fois d'inscrire régionalement cette revue nationale et de la redéployer par de nouveaux supports vers un public plus large et international.

Rappel du contexte scientifique et des enjeux éditoriaux

6 La revue Techniques \& culture correspond à un projet éditorial lancé en 1983 par Robert Cresswell (son directeur actuel) et un collectif de chercheurs en sciences sociales embarqués dans un débat intellectuel exigeant sur les techniques, l'économie, la culture matérielle et le social dans les années 1970 et 1980. Ce groupe de chercheurs, ouvert et sensible aux dimensions sociales et culturelles des techniques, sut, face aux courants dominants de l'anthropologie culturelle des années 1970, développer cette revue interdisciplinaire originale et ouverte tant aux aspects empiriques que théoriques d'étude des techniques. Elle fut d'abord un lieu de confrontation d'idées et de méthodes en des temps où l'épistémologie, le terrain, le matériel, le symbolique et l'économique bavardaient difficilement autour d'une même table. Nous ne rappellerons pas la mythique césure de l'anthropologie entre Levi-Straussiens et Leroi-Gourhaniens, ni les ruptures entre les divers courants matérialistes de l'anthropologie, ou entre l'anthropologie et l'histoire, tant elles semblent désormais datées et peu adéquates.

7 Les temps ont changé, les débats se sont apaisés ou reconfigurés ailleurs, mais, entretemps les disciplines des sciences sociales se sont spécialisées, technicisées et souvent cloisonnées. Dans le panorama actuel de la recherche, les sciences de l'homme et de la société ont de plus en plus de difficultés à tenir une position ferme tant elles sont prises entre deux tendances, celle qui s'observe dans un relativisme « mou », celui des Cultural Studies ou celui de l'essayisme psychologique ou philosophique à la française et celle, tout aussi répandue, d'un matérialisme positiviste (emprunté aux sciences de la nature) à très fort impact actuel sur les SHS. Ajoutons à ce constat par trop schématique que les programmes épistémiques et empiriques élaborés tout au long des traditions européanistes de recherches, tant en sociologie qu'en anthropologie ou en histoire, ont quelques difficultés à s'exprimer dans les cadres utilitaristes et brefs des politiques actuelles de recherche.

8 Face à cette polarisation «relativisme/matérialisme» improductive, la revue Techniques \& culture dispose d'atouts remarquables (ses objets médians de réflexion, son héritage intellectuel, son interdisciplinarité, son ouverture thématique, etc.) qu'il importe plus que jamais d'afficher et d'amplifier, tant pour des raisons heuristiques que politiques.

9 Nous observons aujourd'hui un retour sans précédent du monde matériel et de la matérialité, dans les recherches anglo-saxonnes (cf. la revue anglaise Journal of Material 
Culture) ou dans les grands projets muséographiques français (musée du quai Branly ou musée des Civilisations de l'Europe et de la Méditerranée). Or, ce retour du matériel se fait bien souvent « hors-sol », comme les tomates, belles, lisses mais sans goût, texture ni odeur, autrement dit, ce retour se fait sans les techniques, sans le social, l'économique ni les hommes et les femmes qui vont avec. L'idéel et le matériel sont bien souvent vidés de leur substance.

$10 \mathrm{Au}$ passage, rappelons une banalité : une science sociale n'est sociale que si elle contextualise, historicise, théorise des faits sociaux, qu'elle cadre, compare et coordonne, sinon elle ne fait que "taxidermer » ou reproduire un discours dominant : que ce soit les objets, les arts, les sociétés ou les idées. Le rôle d'une revue et des programmes de travail nationaux et internationaux sur lesquels elle s'adosse doit au contraire faire percuter ensemble idées et terrains, histoires de vies et modèles d'intellection, puiser aux expériences de la recherche en train de se faire, en un mot, initier plutôt que suivre.

11 La revue qui revisiterait ses meilleurs écrits, et qui avancerait un projet ambitieux de réintroduction des objets, des techniques et des dynamiques sociales et culturelles au milieu de la "primitivité ", de l'esthétisation et du relativisme actuels mais aussi au milieu des différents courants tendant à naturaliser le social, n'aurait pas seulement l'écoute et l'estime de ses pairs, mais aussi celle d'un public de plus en plus averti et sur sa faim en matière d'ailleurs et d'autrui, de distinction humaine, pour peu, bien sûr, que l'on se donne les moyens d'être lus par un plus grand nombre.

12 Lié à ce dernier aspect, nous aimerions également rappeler une autre banalité : un champ de recherche dynamique ne craint pas de s'exposer, de se confronter aux autres courants de pensée et d'être largement diffusé. Il entre d'ailleurs dans les obligations des chercheurs de diffuser leurs recherches plus loin que vers leurs pairs. La réussite de certaines revues de sciences humaines en France devrait, à titre d'exemple, nous faire réfléchir de façon fine au lectorat actuel et potentiel de Techniques \& culture. Il n'est bien sûr pas nos visées de faire une revue de kiosque, mais, diffuser de façon large, dans les librairies, les musées ou les centres culturels en France et à l'étranger, nous semble cadrer idéalement avec le profil de la revue telle que nous l'envisageons. En bref, à ce jour, doit-elle être lue par d'autres lecteurs que les seuls étudiants et chercheurs en archéologie, anthropologie ou histoire ? La réponse est évidemment positive. À nous de définir comment, au cours de l'année 2008.

Les grandes lignes éditoriales envisagées

13 Voici, de façon résumée, les quelques orientations que nous souhaitons développer dans les mois à venir.

Une revue interdisciplinaire sur les rapports entre technique et culture

Les techniques sont par essence transversales et ne peuvent s'appréhender correctement qu'à l'aide de plusieurs lentilles disciplinaires. De même, «la » ou " les » cultures comme objets fondateurs res aspects des sociétés, elles jouent comme des révélateurs originaux des relations et des dynamiques sociales. Ce qui signifie en conséquence que ni les techniques ni la culture ou «les» cultures ne peuvent constituer une fin en soi. Pour le dire autrement, la «technologie» -fût-elle «culturelle »- comme discipline unificatrice serait utopique en ce début de XXI ${ }^{\mathrm{e}}$ siècle.

L'état des acquis et des malentendus des études sur les relations entre techniques et cultures pourrait être fait, par exemple, en reprenant les textes fondateurs ou marquants de la revue et en les mettant en miroir avec les textes actuels portant sur les 
mêmes sujets ou des sujets similaires. Ce «best of » des textes passés et sub-actuels sur les rapports entre « techniques et cultures » est encore à réaliser, mais nous semble un des chantiers épistémiques transversaux possibles pour cette revue. Nous nous y attacherons en 2009 avec pour objectif une anthologie (ou "reader») sur l'école française de technologie culturelle, vraisemblablement sous format électronique et papier.

Faire le point sur l'état des recherches sur les techniques signifie également sortir du débat de spécialistes, et en premier lieu, d'écrire pour son voisin d'Amérique latine, d'Océanie ou... de discipline, donc pour un archéologue, d'exposer et d'écrire pour être compris d'un sociologue, ou, pour un anthropologue, de sortir des « entendus » propres à son champ.de l'anthropologie sortent du seul registre de compétence de la discipline et alimentent de nos jours les réflexions d'innombrables courants et disciplines des sciences humaines. Le rééquilibrage et la représentation de disciplines autres que l'anthropologie au sein du comité de rédaction et des publications à venir s'opèrent donc dans cette perspective.

Les techniques exemplifient des médiations entre la nature (physique et construite) et les dispositifs culturels extrêmement variés des sociétés humaines et pré-humaines. Les techniques (les objets, gestes, programmes, etc.) constituent bien souvent des accès privilégiés à d'aut

Une revue principalement thématique fondée sur des journées scientifiques

La revue n'est aujourd'hui plus organiquement rattachée à un laboratoire du même nom et souhaite orienter ses choix éditoriaux sur la base d'un dialogue collectif qui s'appuie sur des enseignements et des journées régulières de rencontres scientifiques qui permettront de faire émerger les thèmes les plus innovants en matière de techniques et de cultures. Ce qui signifie aussi de se fonder davantage sur l'actualité de la recherche (les grands programmes de terrain en cours, les colloques les plus originaux en matière de recherche dans le domaine, etc.).

Toutes sortes de thématiques sont par conséquent envisageables à partir du moment où elles entrent dans un format construit, comparatif et interdisciplinaire (les articles hors thème pouvant toujours être présentés en varia). Nous n'oublierons pas non plus de réaffirmer l'axe un peu négligé ces dernières années d'ouverture aux objets contemporains, du low-tech au high-tech, des traditionnelles questions sur les rythmes d'évolution à celles du genre des techniques, celles développées par le courant des usages ou celles de l'ethnographie cognitive.

20 Passer d'une revue semestrielle -et souvent avec des numéros doubles- à une revue thématique, plus varia, paraissant trois fois l'an nous semble le but à viser, quitte à diminuer la taille des volumes mais à assurer une parution régulière et fréquente sur les portoirs des bibliothèques, des librairies ou des musées.

Une revue à visée internationale

21 Une plus grande ouverture à l'interdisciplinarité, peut et doit s'accompagner d'une confrontation et d'une diffusion internationale, les approches et disciplines ne se traduisant pas toujours aisément d'un continent à un autre. Les champs disciplinaires ont en effet souvent fini par s'isoler (et parfois réinventer la roue -avec un nouveau vocabulaire- chacun de leur côté). Nous pensons en France au courant de la sociologie des usages qui a ignoré celui de l'analyse des activités tel qu'il a été développé en 
anthropologie et qui s'exprimerait par exemple bien différemment dans un contexte canadien, francophone ou anglophone.

Les revues Réseaux, Publics et musées, Journal of Material Culture, Techniques \& culture, Terrain, Anthropologie et sociétés, œuvrent parfois sur des objets proches mais selon des procédures et traditions scientifiques qui gagneraient à être débattues au sein de la revue (à propos de la notion de « culture matérielle » par exemple).

Diffuser largement les recherches francophones sur les rapports entre techniques et cultures signifie aussi s'engager et tenter de peser sur les problématiques standards de la recherche actuelle. Les autoroutes thématiques: identity, gender, minorities, postcolonial studies, evolutionary studies, etc. de l'anthropologie, pour n'en citer que quelquesunes, sont incontournables, mais sont-elles les plus innovantes ou encore celles sur lesquelles il convient de parier si l'on souhaite développer une voie spécifique aux sciences humaines et sociales francophones? Nous pouvons faire d'autres paris.

Selon nous, la revue peut et doit donc être conçue comme un observatoire et un lieu de prospection et d'expérimentation. Elle sera diffusée sur Internet en anglais (et en français) afin de donner à lire les différents travaux francophones au monde académique anglophone, et en retour, de nous confronter à cet univers globalisé. Nous procéderons par ailleurs à la numérisation de l'ensemble des numéros de la revue depuis ses origines, de 1976 aux numéros déjà en ligne sur le site Revues.org (http:// tc.revues.org/).

Une revue utilisant au maximum la photographie et le graphisme

Entre les revues d'exploration ou les catalogues d'art primitif sur papier glacé et les revues austères, théoriques et lettrées des ethnologues ou historiens, il existe un champ immense d'action dans lequel placer la revue Techniques \& culture, à condition bien sûr, d'y prêter une certaine attention et d'allouer les moyens qu'il convient.

D'où le projet d'une revue savante avec une nouvelle maquette au design et au format étudiés en fonction des contenus et des lectorats visés, mais, au premier chef, amplement illustrée de photos couleur et noir et blanc, de dessins, de graphes de haute qualité. Les images devront pouvoir être intégrées et commentées avec la même attention que l'on accorde habituellement au texte, c'est ce qui fera aussi la différence avec les ouvrages d'illustration ethnographiques ou à thèmes "sociaux" qui se multiplient aujourd'hui mais qui ne reproduisent, bien souvent, sous des formes esthétisantes, que des clichés éculés sur l'autrui, la nature ou les « arts dits premiers ».

L'exposé photographique pourra appuyer la thèse scientifique, l'étayant et amenant un plus grand nombre de lecteurs à saisir les propos. Au-delà de ce parti pris de vulgarisation, il y a aussi l'idée qu'il importe, et cela plus précisément dans une revue qui traite aussi de techniques et d'objets, de donner à lire et à voir, des hommes en activité, des hommes en société et non des hommes "empaillés ", esthétisés, hors du temps. Le design graphique de la revue sera pensé en fonction de ce type de position politique et non en fonction de tel ou tel « style » en vogue sur le net ou dans les revues dites artistiques.

Des thèmes transversaux, entre empirie et théorie

Si nous avons avancé un peu rapidement ce contraste entre revue littéraire, savante et catalogues trop vite faits, c'était pour faire bref. Il existe bien sûr toute une gamme intermédiaire de productions écrites et graphiques à laquelle cette revue de science ne peut prétendre, mais, si l'on compare la classe des revues à laquelle elle appartient 
(L'Homme, Études rurales, Ethnologie française, Terrain, Gradhiva, Anthropologie et sociétés pour ne prendre que les revues francophones d'anthropologie) nous opterons plutôt pour une formule thématique « et » interdisciplinaire.

Dès le numéro 50, la revue se présentera sous la forme d'un numéro à thème, ce thème transversal étant abordé par différentes disciplines et suivi d'un ensemble d'articles variés. Le lecteur aura donc «l'attendu » (le thème fondé sur des études de terrain pris selon différents points de vue disciplinaires et problématisé par un responsable du comité de rédaction et un spécialiste extérieur à la revue) et "l'inattendu ", avec des varia et une RAB ("Rubrique à Brac») avec des "à propos" et pourquoi pas "des objets du semestre " que nous inviterions chercheurs et amateurs à décrire et commenter. Les actualités et comptes rendus trouveront plus idéalement leur place sur le site Web de la revue.

La revue privilégiera, thème par thème, l'activité scientifique dans toute sa chaîne de production, de l'observation à la restitution la participation de philosophes, de sociologues, d'archéologues, de muséologues ou de spécialistes des sciences de la communication, favorisant ce redéploiement le travail sous forme de journées critiques et interdisciplinaires assurant une exigence scientifique de qualité.

31 À titre d'exemple, pour donner "matière à penser ", comme l'écrivent certains de nos collègues, voici quelques thèmes envisagés pour les numéros à venir : « les natures de l'homme " "nouveaux regards sur les dynamiques culturelles " "hommes, objets et musées de sociétés » " savoirs et techniques pastorales " « l'objet en procès » " gestes et actions élémentaires sur la matière : quel bilan, quels projets?» "le rendez-vous français manqué de l'ethnoarchéologie?» "l'efficacité technique, entre biologie et culture» "arts, artisans, artistes» "objets et techniques du culte» "dispositifs immatériels» «communautés de pratiques» «affordances et agencies» « usages photographiques et "révolution" numérique " « cultures automobiles » ...

Ces quelques thèmes ont pour certains déjà trouvé preneur, pour les autres, à nous de les construire, déconstruire, reconstruire, et bientôt, de vous les donner à lire.

33 Nous espérons que ces propositions inédites (thématiques co-élaborées, graphies et mise en ligne), amplifieront avantageusement la formule actuelle, sans trop la changer toutefois. En étant fermes et systématiques dans leurs applications, ces propositions devraient pouvoir assurer une meilleure audience nationale et internationale à la revue.

En attendant ces changements, nous sommes heureux, pour les auteurs en souffrance et les lecteurs en attente, de vous livrer avec ce numéro 48-49 -le dernier de cette série -, un ensemble de textes originaux sur différents terrains traditionnels et contemporains en anthropologie et en histoire des techniques. Nous les avons organisés en différents moments, intitulés "Temps, corps, techniques et esthétique ", et cherché le mieux possible à composer textes et images. Ils donnent une idée de la diversité des recherches sur les techniques que nous tenterons d'ouvrir et de structurer encore plus fortement avec les numéros à venir. 


\section{AUTEURS}

FRÉDÉRIC JOULIAN

EHESS frederic.joulian@ehess.fr

ROBERT CRESSWELL 